\title{
Voyage from fiction to reality: organs-on-chips in the Service of Science and Medicine
}

\author{
De la ficción a la realidad: órganos-en-chips al Servicio \\ de la Ciencia y la Medicina
}

\author{
Marina Macías Silva*
}

Organs-on-chips are micrometrical-scale manufactured devices. They have been described as biomimetic systems purporting the main function of achieving preservation of the functional unit of a living organ in a tri-dimensional structure (3D). Thus, biology-inspired technology has led to the fabrication of the devices which consist in micro-luidic 3D minute channels, manufactured with a clear polymeric material lined with cells. These devices seek to replicate important aspects of an organ, such as $3 D$ microarchitecture which takes place due to distribution in space of different tissues, functional inter-faces between tissue-tissue and organs' mechanical and biochemical complex microenvironments. Thus, these devices known as organs-on-chips recapitulate the complexity of live organs with respect to their architecture, microenvironment and physiology.

Cell processes which have been studied using this type of devices are very diverse; among them we can count the following: cell growth and death, cell and tissue morphology, differentiation, cell motility and contractility, function of tissue barriers, metabolism, secretion and oxidative stress. Moreover, research has been conducted on some normal and pathological biological processes such as epithelial-mesenchymal transition (EMT), cardiac contraction, drug metabolism, vasculogenesis, tumor angiogenesis, cancer cell invasion and metastasis, effectiveness and side effects of some drugs and mechanobiology among others.

Mechanobiology is a process by which cells transform mechanical signals into biological responses. This process is initiated through varied mechanical stimuli such as laminar stress, interstitial flow, or tension exerted by the extra-cellular matrix (ECM). These stimuli cause different biological responses in cells or tissues, such as, for example, gene differential expression, protein secretion or cell migration. It is common for these biological responses to involve changes in cells' intrinsic mechanical properties, such as generation of changes in the cytoskeleton rigidity. Moreover, in certain cases, mechanotransduction involves feedback processes, that is to say that intracellular changes can modify external mechanical signals; a feedback case takes place when, for example a laminar flow stress (external mechanical signal) causes cell polarization or elongation (biological response), this leads to alterations in fluid flow and concurrently to an alteration of laminar stress which is exerted on the epithelial monolayer. In order to research processes such a mechanobiology, a specific sort of cell cultures is being developed, which use all the advances of micro-manufacturing and micro-fluidic techniques, in order to control cell micro-environment and mimic physiological context. Organ-on-chip type devices are at the forefront of this type of technological developments. In these devices, the simplest possible model is created, so as to recapitulate physiological responses under study. It is worth mentioning that development of these technologies requires the joint effort of engineers, physicists, biologists, computational biologists and physicians, so as to be able to implement methodologies which will allow to capture essential components of the organs physiology under study.

Another aspect that should be emphasized are the advantages provided by 3D culture models, which, differing from $2 \mathrm{D}$ cultures allow to recapitulate in vivo all those complex interactions regulated by several factors such as cytokines, nutrients, growth factors, hormones, extracellular matrix and intercellular joints. In organs-on-chips it is possible to control from

\footnotetext{
* Cellular Physiology Institute, National Autonomous University of Mexico (UNAM).
}

This article can be read in its full version in the following page: http://www.medigraphic.com/facultadodontologiaunam 
substrate to cultivate cells up to movement of fluids in the culture to thus reproduce mechanical signals induced by physiological flows ( for example blood and interstitial flows), as well as tissue deformation (for example respiration, peristalsis, and heartbeat, and also including stress due to laminar flow, tension, compression and torsion. Thus, in recent years, these biomimetic systems have been used to establish models able to recapitulate structural and functional complexity of some human organs such as liver, heart, lung, kidney brain and bone. Presently, scientists have also begun to develop multiorgans, able to replicate sequential processes, for example, those that could influence drug activity in the body; thus, these new systems called body-on-a-chip are designed to better mimic complexity at the level of an organ as well as organ-organ interactions.

Within the realm of technological development of organs-on-chips, one of the encountered technical challenges is related to the choice of type of materials used in their manufacture, among which polycarbonate, polystyrene, and a type of silicon called PDMS (Polymethylsiloxane) are found. These materials are used as culture substrate, nevertheless, their physical and chemical properties unfortunately do not mimic in vivo extracellular matrix. PDMS is one of the most frequently used materials for the manufacture of these devices, although its use implies many advantages, some deficiencies have been observed, such as the ability to absorb small-sized molecules, which would decrease drugs' effective concentrations and diminish activity in cultures carried out in microdevices. Some studies have shown that comparison of cell cultures undertaken according to traditional methods with cultures performed in microfluidic devices reveals significant differences with respect to cell proliferation and cell metabolic activity (oxygen consumption, protein synthesis, etc.). Therefore, techniques are being implemented presently to chemically modify PDMS surfaces, and at the same time the use of alternative materials is being explored to thus establish more favorable cell culture conditions in this type of microdevices, avoiding therefore all changes in the phenotype or cell context.

On the other hand, work is being undertaken in the field of integration or organs-on-chips into bioanalytical platforms including sensors and miniaturized optics in order to achieve high-resolution biochemical analyses with much reduced amounts of sample. One of the most promising applications of organs-onchips technology is to serve as a type of additional pre-clinical analysis in animal studies, in order to generate necessary predictions before undertaking clinical studies in humans. Presently, one of the challenges faced by the pharmaceutical industry is to be able to decrease costs and potentiate research and development of new drugs with clinical use. Therefore, these biomimetic systems can be used as specialized in vitro models to facilitate mechanistic research and pharmacological modulation of many biological processes. In the future, these devices might be used in personalized (custom-made) medicine and in clinical studies where the patient's own cells might be cultured in these devices, in a sort of human-on-a-chip to thus study use of drugs' safety, their effectiveness and side effects which could occur in any given subject. It is worth mentioning that advances in this type of developments will depend not only on scientists' efforts, but also on agreements established among academic institutions, industry and government. It is thus expected that very soon organ-on-chip body-onchip and even organism-on-chip devices will become technologies with greater number of applications in biomedicine.

\section{REFERENCES}

1. Bhatia SN, Ingber DE. Microfluidic organs-on-chips. Nat Biotechnol. 2014; 32 (8): 760-772.

2. Derkus $B$. Applying the miniaturization technologies for biosensor design. Biosensor Bioelectron. 2016; 79: 901-913.

3. Esch EW et al. Organs-on-chips at the frontiers of drug discovery. Nat Rev Drug Discov. 2015; 14 (4): 248-260.

4. Hamon M, Hong JW. New tools and new biology: recent miniaturized systems for molecular and cellular biology. Mol Cells. 2013; 36 (6): 485-506.

5. Hautefeuille M. Jefe del Laboratorio Nacional de Sistemas Biomiméticos para Diagnóstico y Terapia, de la Facultad de Ciencias de la UNAM. LANSBioDyT: Available in: https://sites. google.com/a/ciencias.unam.mx/lansbiodyt/home

6. Materne EM et al. The multi-organ chip - a microfluidic platform for long-term multi-tissue coculture. J Vis Exp. 2015; 98: e52526.

7. Paguirigan AL, Beebe DJ. From the cellular perspective: exploring differences in the cellular baseline in microscale and microfluidic cultures. Integr Biol (Camb). 2009; 2: 182-195.

8. Perestrelo AR et al. Microfluidic organ/body-on-a-chip devices at the convergence of biology and microengineering. Sensors (Basel). 2015; 12: 31142-31170.

9. Polacheck WJ et al. Microfluidic platforms for mechanobiology. Lab Chip. 2013; 13: 2252-2267.

10. Vázquez-Victorio G et al. Chapter 9: GPCRs and actincytoskeleton dynamics. Methods in Cell Biology. 2016; 132. Elsevier, Inc. (En prensa).

Mailing address:

Marina Macías Silva

E-mail: mmacias@ifc.unam.mx 